\title{
RD3, the protein associated with Leber congenital amaurosis type 12, is required for guanylate cyclase trafficking in photoreceptor cells
}

\author{
Seifollah Azadi, Laurie L. Molday, and Robert S. Molday ${ }^{1}$ \\ Department of Biochemistry and Molecular Biology and Department of Ophthalmology and Visual Sciences, Centre for Macular Research, University of British \\ Columbia, Vancouver, BC, Canada V6T 1 Z3
}

Edited by Jeremy Nathans, The Johns Hopkins University, Baltimore, MD, and approved October 13, 2010 (received for review July 17, 2010)

\begin{abstract}
Guanylate cyclases, GC1 and GC2, are localized in the light-sensitive outer segment compartment of photoreceptor cells, where they play a crucial role in phototransduction by catalyzing the synthesis of CGMP, the second messenger of phototransduction, and regulating intracellular $\mathrm{Ca}^{2+}$ levels in combination with the cGMP-gated channel. Mutations in GC1 are known to cause Leber congenital amaurosis type 1 (LCA1), a childhood disease associated with severe vision loss. Although the enzymatic and regulatory properties of guanylate cyclases have been studied extensively, the molecular determinants responsible for their trafficking in photoreceptors remain unknown. Here we show that RD3, a protein of unknown function encoded by a gene associated with photoreceptor degeneration in humans with Leber congenital amaurosis type 12 (LCA12), the $r d 3$ mouse, and $r c d 2$ collie, colocalizes and interacts with GC1 and GC2 in rod and cone photoreceptor cells of normal mice. GC1 and GC2 are undetectable in photoreceptors of the $r d 3$ mouse deficient in RD3 by immunofluorescence microscopy. Cell expression studies show that RD3 mediates the export of GC1 from the endoplasmic reticulum to endosomal vesicles, and that the $C$ terminus of GC1 is required for RD3 binding. Our results indicate that photoreceptor degeneration in the $r d 3$ mouse, $r c d 2$ dog, and LCA12 patients is caused by impaired RD3-mediated guanylate cyclase expression and trafficking. The resulting deficiency in cGMP synthesis and the constitutive closure of cGMP-gated channels might cause a reduction in intracellular $\mathrm{Ca}^{2+}$ to a level below that required for long-term photoreceptor cell survival.
\end{abstract}

membrane trafficking | photoreceptor degeneration | vision | calcium homeostasis | retinal disease mechanisms

eber congenital amaurosis (LCA) is a heterogeneous group of Linherited retinal degenerative diseases that cause severe blindness at birth or within the first year of life. LCA, most commonly inherited as an autosomal recessive disorder, is typically characterized by progressive changes in the appearance of the retina, absent or markedly reduced electroretinography (ERG) responses, and nystagmus, in addition to impaired vision (1). To date, mutations in 14 different genes have been associated with various forms of LCA. These genes encode various proteins that have been implicated in such diverse cellular pathways as phototransduction, vitamin A metabolism, protein assembly, ciliary transport, photoreceptor development and morphogenesis, guanine nucleotide synthesis, and outer segment phagocytosis (1).

$G U C Y 2 D$, which encodes guanylate cyclase 1 (GC1), was the first gene associated with LCA (2). GC1 plays a crucial role in phototransduction by catalyzing the synthesis of cGMP in rod and cone photoreceptors. Photobleaching of rhodopsin in rod outer segments or of cone opsin in cone outer segments activates the $G$ protein-coupled visual cascade, leading to the activation of phosphodiesterase, hydrolysis of cGMP, closure of cGMP-gated channels to the influx of $\mathrm{Na}^{+}$and $\mathrm{Ca}^{2+}$, and hyperpolarization of the cell (3). After photoexcitation, recovery of the cell to its dark state occurs through the inactivation of opsin, shutoff of the visual cascade, and resynthesis of cGMP by the activation of membrane- bound GCs via guanylate cyclase activating proteins GCAP1 and GCAP2. The finding that mutations in the GUCY2D gene cause Leber congenital amaurosis type 1 (LCA1) underscores the importance of $\mathrm{GC1}$ in the function and survival of rod and cone photoreceptors. Rod photoreceptors also contain another, less abundant retinal-specific GC isoform known as GC2, which is encoded by the $G U C Y 2 F$ gene (4); however, to date, this gene has not been implicated in any retinal degenerative disease.

Proteins involved in phototransduction are synthesized in the inner segment of photoreceptors and subsequently translocated through the cilium to the outer segments. The trafficking of rhodopsin in rod photoreceptor cells has been studied in some detail (5-7); however, the molecular determinants important to the trafficking of most other phototransduction proteins, including the membrane GCs GC1 and GC2, have not yet been determined.

The $r d 3$ mouse is one of the earliest identified naturally occurring mouse strains with severe early-onset retinal degeneration (8). The retina of homozygous $r d 3$ mice develop normally through postnatal day 14, but subsequently undergo progressive photoreceptor degeneration such that few rod or cone cells remain after 2-4 mo $(9,10)$. The gene responsible for photoreceptor degeneration in the $r d 3$ mouse encodes a 195-aa protein of unknown subcellular localization and function that is highly expressed in the retina $(10,11)$. Sequence analysis indicates that RD3 contains putative coiled-coil domains at amino acids 22-54 and 115-141 and several casein kinase II and protein kinase $\mathrm{C}$ phosphorylation sites. A homozygous c.319C $\rightarrow$ T substitution in exon 3 of the $R d 3$ gene (formally the C1orf36 gene) results in an unstable truncated protein lacking the $\mathrm{C}$-terminal 89 amino acids. A mutation in the human $R D 3$ gene that also causes premature protein truncation is responsible for Leber congenital amaurosis type 12 (LCA12), and a frameshift mutation in canine $R D 3$ resulting in an altered Cterminal protein sequence is associated with rod-cone dysplasia type 2 ( $r c d 2)$ in collie dogs $(10,12)$. These previous studies underscore the importance of RD3 in photoreceptor cell survival, but do not provide insight into the function of RD3 in photoreceptor cells or the mechanism through which mutations in RD3 cause photoreceptor degeneration.

Recently, we carried out a mass spectrometry-based proteomic analysis of isolated photoreceptor outer segments to identify proteins that might play crucial roles in photoreceptor structure, function, protein trafficking, and inherited retinal degenerative diseases (13). RD3 was one of the retinal-specific proteins identified in the proteomic dataset. In the present study, we show that RD3 colocalizes and binds to GC1 and GC2 and plays a crucial

Author contributions: S.A., L.L.M., and R.S.M. designed research; S.A. and L.L.M. performed research; S.A., L.L.M., and R.S.M. analyzed data; and R.S.M. wrote the paper. The authors declare no conflict of interest.

This article is a PNAS Direct Submission.

${ }^{1}$ To whom correspondence should be addressed. E-mail: molday@interchange.ubc.ca.

This article contains supporting information online at www.pnas.org/lookup/suppl/doi:10 1073/pnas.1010460107/-/DCSupplemental. 
role in their stable expression and membrane trafficking in rod and cone photoreceptors. These findings provide insight into the molecular mechanism responsible for photoreceptor degeneration in LCA12 patients and animals deficient in the RD3 protein.

\section{Results}

Subcellular Localization of RD3 in Photoreceptor Cells. To determine the subcellular localization of RD3 and identify interacting proteins, we generated specific monoclonal and polyclonal antibodies to the C-terminal region of RD3. Both antibodies labeled the 23-kDa RD3 protein in retinal extracts of WT mice, which was absent in retinal extracts of homozygous $r d 3$ mice (Fig. $1 A$ ). The subcellular distribution of RD3 in WT mouse retinal sections was determined by immunofluorescence labeling for confocal scanning microscopy (Fig. 1B). Intense immunostaining was evident in rod and cone outer segments of 21-d-old WT mice, with only faint staining detectable in the inner segments and synaptic region. Retinal sections of 21-d-old homozygous $r d 3$ mice (4Bnr, albino strain), which have $\approx 80 \%$ of the normal complement of photoreceptors cells (9), were not labeled, confirming the specificity of the anti-RD3 antibody and the absence of the full-length RD3 protein (Fig. 1B). Localization of RD3 to photoreceptor outer segments is consistent with our earlier proteomics study showing the presence of RD3 in outer segment preparations by mass spectrometry (13) and biochemical subcellular fractionation of a mouse retinal homogenate carried out as part of the present study (Fig. S1).

RD3 Binds to GC1 and GC2. To begin to define the role of RD3 in photoreceptors, we identified proteins that interact with RD3 by coimmunoprecipitation along with mass spectrometry and Western blotting. A detergent-solubilized retinal extract from WT mice was applied to an immunoaffinity column consisting of the $\mathrm{Rd} 3$ 9D12 monoclonal antibody coupled to Sepharose beads. After the unbound proteins were removed, bound proteins were eluted with SDS and digested with trypsin for analysis of peptides by liquid chromatography-tandem mass spectrometry (LC-MS/MS). Photoreceptor GC1 and GC2 (also known as GC-E and GC-F, respectively) and RD3, along with other proteins, were identified with a high level of confidence (Table S1).
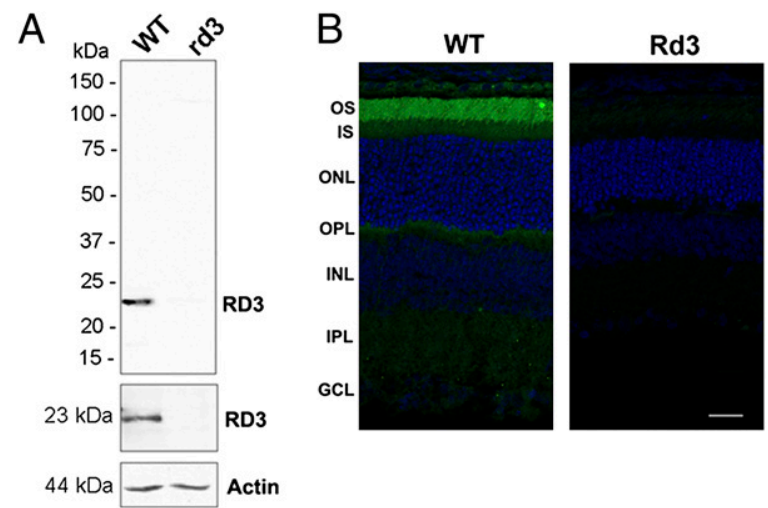

Fig. 1. Expression and localization of RD3 in mouse retina. $(A)$ Western blot analysis of 21-d-old WT and homozygous $r d 3$ mouse retinal membrane extracts labeled with the Rd3-9D12 monoclonal antibody (Top), a polyclonal antibody to RD3 (Middle), and an anti-actin antibody as a loading control (Bottom). RD3 was present in the WT membrane extract, but absent in the $r d 3$ membrane extract. $(B)$ Confocal images of 21-d-old WT and $r d 3$ mouse retinal cryosections labeled with the purified polyclonal antibody to RD3 (green) and counterstained with DAPI nuclear stain (blue). RD3 is localized primarily to the rod and cone outer segments. OS, outer segment; IS, inner segment; ONL, outer nuclear layer; OPL, outer plexiform layer; INL, inner nuclear layer; IPL, inner plexiform layer; GCL, ganglion cell layer. (Scale bar: $20 \mu \mathrm{m}$.)
Because LC-MS/MS is extremely sensitive and does not give quantitative information on the relative protein abundance, we further analyzed the input, unbound, and bound fractions from the anti-RD3 immunoaffinity column on SDS gels and Western blots labeled with antibodies for various outer segment and cytoskeleton proteins. As shown in Fig. 2A, a single 120-kDa band corresponding to the molecular mass of photoreceptor $\mathrm{GC} 1$, the most abundant photoreceptor GC, was observed in the Coomassie blue-stained lane of the bound fraction from the anti-RD3 immunoaffinity column. Western blot analysis confirmed the identity of this band as GC1 and revealed the presence of GC2 with a similar molecular mass. Control experiments using an ABCA4 immunoaffinity column showed that GC1 does not bind nonspecifically to immunoaffinity resins (Fig. S2). The 23-kDa RD3 protein, which was not visible in the Coomassie blue-stained gels, was present in the bound fraction by Western blot analysis (Fig. 2A). A comparison of the input and unbound fraction of the Western blots labeled for GC1 and GC2 indicated that $>50 \%$ of the GC1 and GC2 coimmunoprecipitated with RD3 in the presence of detergent. Although phosphodiesterase (PDE), the cyclic

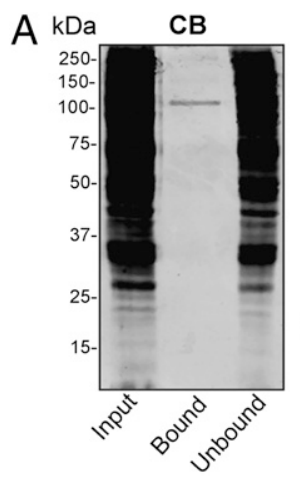

B

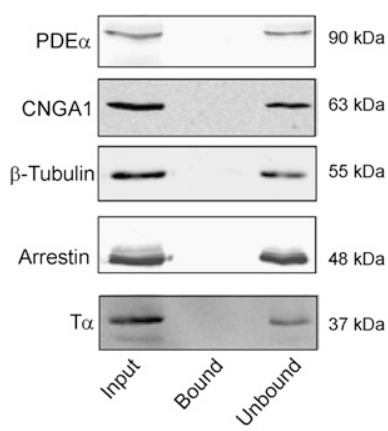

GC1/RD3

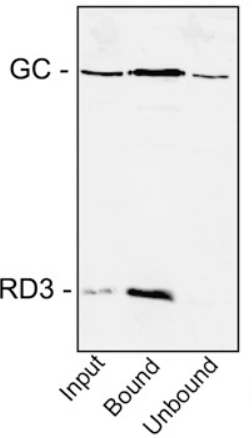

GC2/RD3
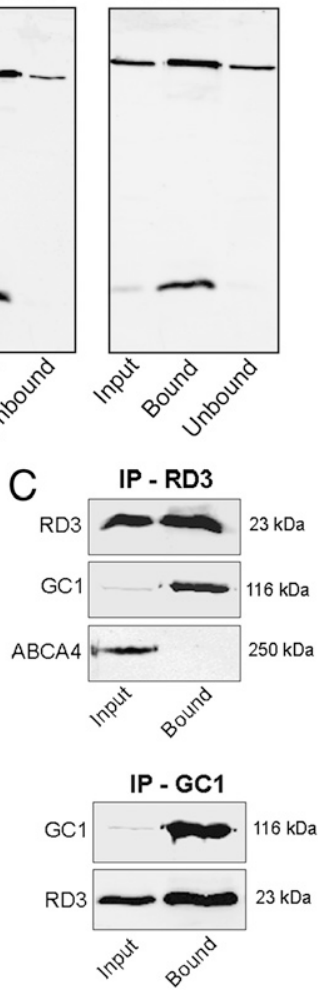

Fig. 2. Coimmunoprecipitation of GC1 and GC2 with RD3. (A) A detergentsolubilized WT mouse retinal extract was incubated with an Rd3-9D12 immunoaffinity matrix. After the unbound protein was removed, the bound protein was eluted from the matrix with SDS for analysis on Coomassie bluestained gels (CB) and Western blots labeled with the Rd3-9D12 antibody against RD3 and polyclonal antibodies against GC1 and GC2 (GC1/RD3 and $\mathrm{GC2} / \mathrm{RD} 3$ ). Approximately $70 \mu \mathrm{g}$ of protein was applied to the input (retinal extract) and unbound lanes. $(B)$ The same Western blots as in $A$, but labeled with antibodies to PDE- $\alpha$, CNGA1, $\beta$-tubulin, arrestin, and transducin (T $\alpha)$. These proteins did not coimmunoprecipitate with RD3. (C) HEK293T cells coexpressing RD3 and either GC1 or ABCA4 as a control were immunoprecipitated on an Rd3-9D12 immunoaffinity matrix (IP-RD3) or an GC1-2H6 matrix (IP-GC1), and the input and bound fractions were analyzed on Western blots labeled for RD3 (Rd3 9D12 antibody), GC1 (GC-2H6 antibody), or ABCA4 (Rim-3F4 antibody). GC1 coimmunoprecipitated with RD3, but ABCA4 did not. 
nucleotide-gated (CNG) channel, arrestin, transducin, and tubulin were detected in the bound fraction by LC/MS-MS (Table S1), they were not observed by Western blot analysis, suggesting that these proteins are minor contaminants of the immunoaffinity procedure (Fig. $2 B$ ).

We further studied the interaction of RD3 with GC1 by coexpression and coimmunoprecipitation studies using HEK293T cells. GC1 specifically coimmunoprecipitated with RD3 from a detergent-solubilized HEK293 cell extract, suggesting that RD3 directly interacts with $\mathrm{GC} 1$ (Fig. $2 C$ ). In reverse experiments, RD3 coprecipitated with GC1 on anti-GC1 immunoaffinity column from both retinal extracts and HEK293T cells coexpressing RD3 and GC1. In control studies, ABCA4, an abundant photoreceptor membrane protein (14), did not coprecipitate with RD3 from either retinal extracts or HEK293T cells coexpressing RD3 and ABCA4, supporting the specificity of the coimmunoprecipitation experiments.

Absence of GC Protein Expression in the Retina of $\boldsymbol{R} d \mathbf{3}$ Mice. We further investigated the role of RD3 in photoreceptor cells by comparing the expression and subcellular distribution of outer segment proteins in retinal tissue of 21-d-old $r d 3$ mice and of agematched WT mice by immunofluorescence microscopy and Western blot analysis (Fig. $3 A$ and $B$ ). Because RD3 interacts with photoreceptor GCs, we first examined the distribution of GC1 and GC2 by confocal scanning microscopy. GC1 and GC2 localized to the outer segment layer of WT mouse retina, with GC1 expressed in both rod and cone outer segments and GC2 present only in rod outer segments, in agreement with previous reports (15-18). In contrast, GC1 and GC2 immunostaining was undetectable in rods and cones of the $r d 3$ mouse retina (Fig. $3 A$ ).

Because the GC-activating proteins GCAP1 and GCAP2 interact with and regulate the enzymatic activities of photoreceptor GCs $(19,20)$, we examined the expression and distribution of GCAP proteins in the retina of $r d 3$ mice. Both GCAP1 and GCAP2 showed reduced expression and mislocalization to the inner segments of photoreceptor cells (Fig. $3 A$ ), similar to that observed in GC knockout mice $(15,16)$.
We also examined the subcellular distribution of other outer segment proteins to determine whether RD3 deficiency affected their expression and trafficking in photoreceptors. Although the expression levels of CNGA1, ABCA4, rhodopsin, peripherin-2, and PDE- $\alpha$ were generally lower in the homozygous $r d 3$ mouse retina, due primarily to the loss of photoreceptor cells and a reduction in outer segment length, the localization of these proteins to outer segments appeared normal (Fig. $3 A$ ).

Western blots of retinal extracts from WT and $r d 3$ mice were labeled for GC1 and GC2 to determine the effect of RD3 deficiency on their level of protein expression. GC1 was undetectable in retinal extracts of $r d 3$ mice, whereas GC2 was detected at a very low level (Fig. $3 B$ ). ABCA4, PDE- $\alpha$, and CNGA1 were present in $r d 3$ extracts at $40-70 \%$ of the levels found in WT extracts, and GCAP1 was present at a significantly reduced level (Fig. S3). We used semiquantitative RT-PCR to examine whether the reduction in GC1 and GC2 expression occurred at the transcriptional level. The level of GC1 and GC2 mRNA expression was similar in the retinas of $r d 3$ mice and WT mice, indicating that GC loss occurred at a protein level.

Effect of RD3 on the Membrane Trafficking of GCs in COS-7 Cells. To further evaluate the role of RD3 in GC membrane trafficking, we expressed RD3 alone and together with GC1 in COS-7 cells and examined their subcellular distribution by immunofluorescence microscopy (Fig. 4). When expressed individually, RD3 and GC1 displayed strikingly different subcellular localizations. RD3 colocalized with the endosomal recycling marker Rab11 in a punctuate staining pattern characteristic of intracellular vesicles. In contrast, GC1 colocalized with calnexin in a perinuclear reticular staining pattern characteristic of endoplasmic reticulum (ER) localization, as reported previously (21). Interestingly, when GC1 was coexpressed with RD3 in COS-7 cells, GC1 was exported from the ER to endosomal vesicles containing RD3 and Rab11. The ability of RD3 to recruit GC1 into intracellular vesicles is specific; even when coexpressed with RD3, ABCA4 localized to intracellular vesicles that did not contain RD3 (Fig. 4). The RD3mediated trafficking of GC1 to intracellular vesicles is consistent with subcellular vesicle localization of other photoreceptor outer
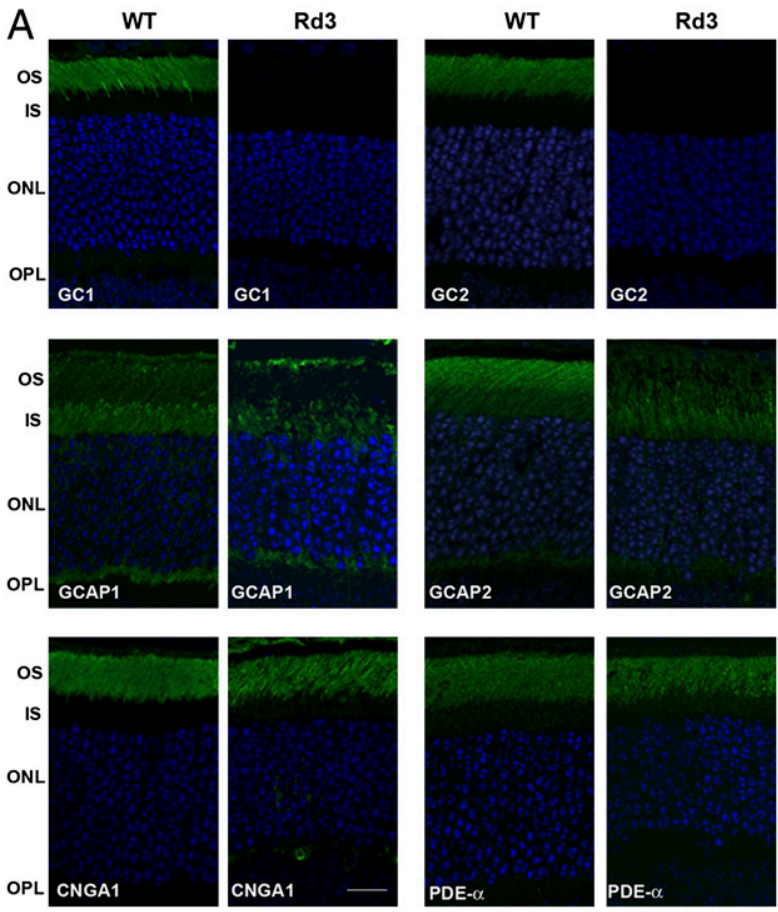
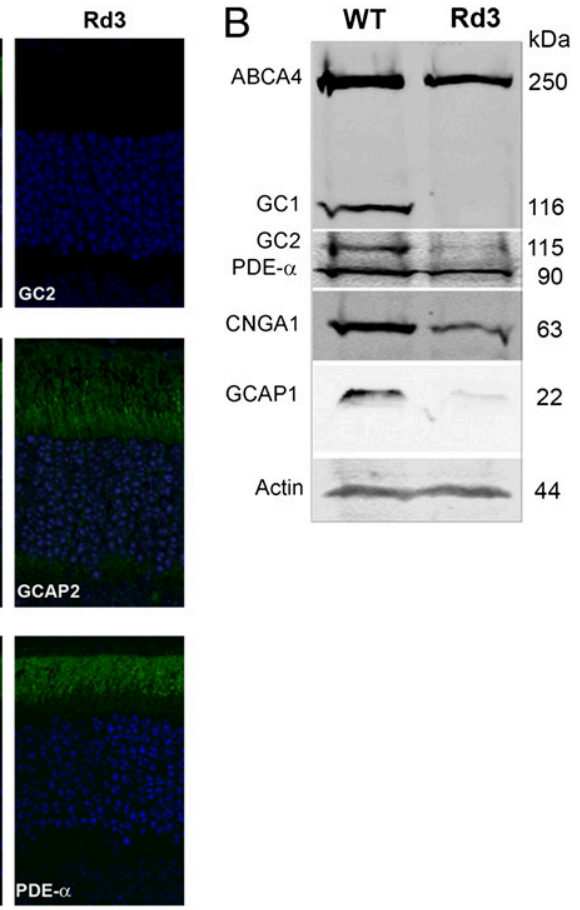

Fig. 3. Expression and localization of GCs and other photoreceptor proteins in WT and $r d 3$ mouse retina by immunofluorescence microscopy and Western blot analysis. $(A)$ Retina cryosections from 21-d-old WT and rd3 mice were labeled with antibodies to GC1, GC2, GCAP1, GCAP2, CNGA1, and PDE- $\alpha$. GC1 and GC2 localized to the outer segments of the WT retina but were undetectable in the $r d 3$ retina. GCAP1 and GCAP2 localized to photoreceptor outer and inner segments of the WT retina but showed reduced expression and mislocalization in photoreceptors of the $r d 3$ retina. CNGA1 and PDE- $\alpha$ localized to the outer segments of both the WT and $r d 3$ retina. Other proteins, including ABCA4, perpherin-2, and rhodopsin, exhibited a normal outer segment distribution in the $\mathrm{rd} 3$ retina. (Scale bar: $20 \mu \mathrm{m}$.) $(B)$ Western blots of retinal extracts from WT and rd3 mice labeled with antibodies to GC1 and GC2 and various outer segment proteins. Actin was used as a loading control. 

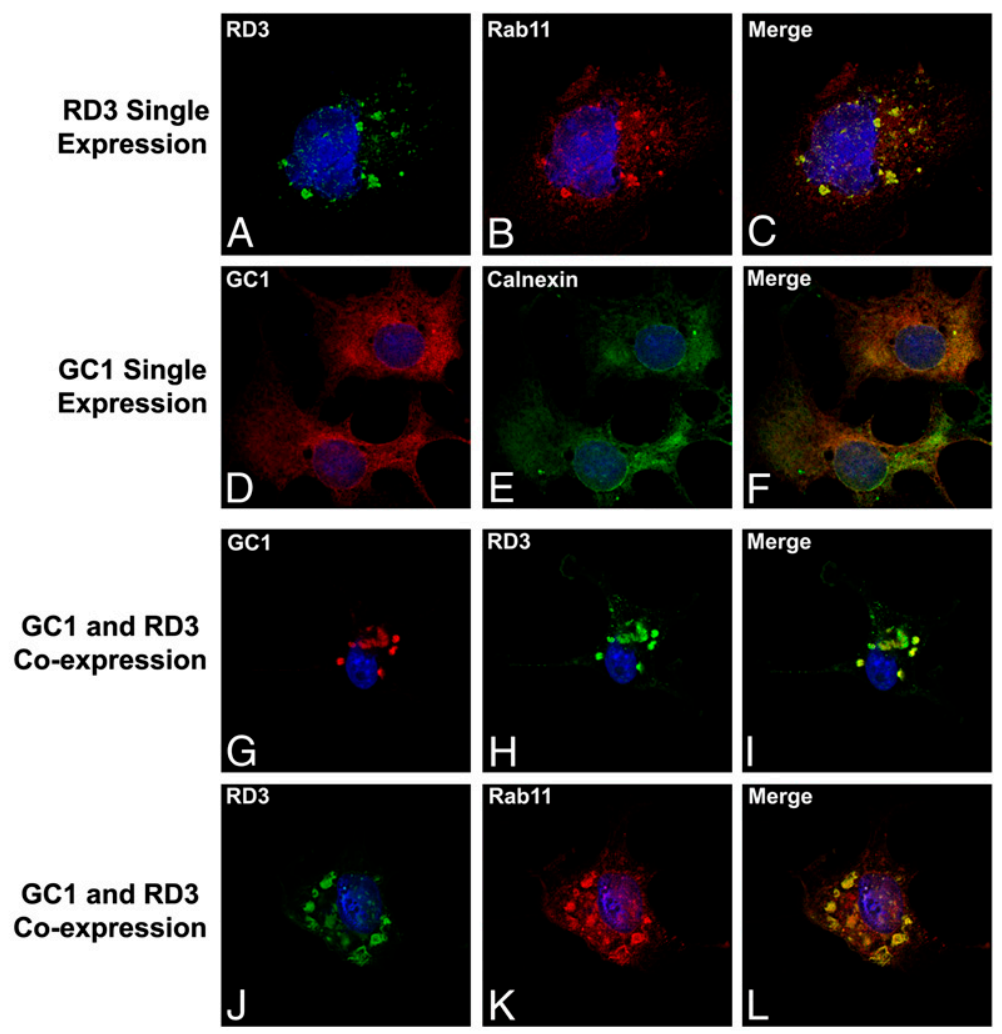

GC1 and RD3 Co-expression
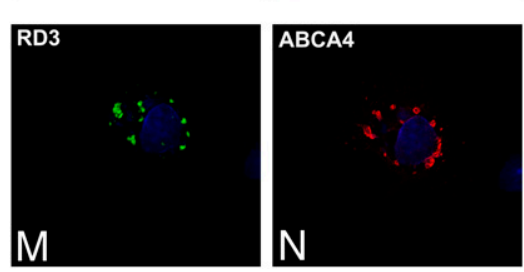

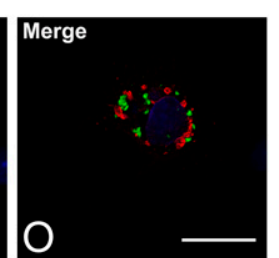

Fig. 4. The effect of RD3 expression on the localization of GC1 in transfected COS-7 cells. Transfected COS-7 cells expressing either RD3 or $\mathrm{GC} 1$ alone or coexpressing RD3 with either $\mathrm{GC} 1$ or ABCA4 were labeled with antibodies to RD3, GC1, ABCA4, and Rab11 or calnexin as indicated. $(A-C)$ RD3 colocalized with Rab11 to intracellular vesicles of RD3-transfected cells. ( $D-F)$ GC1 colocalized with the ER marker calnexin in a pattern characteristic of ER localization in GC1-transfected cells. (G-I) RD3 recruited GC1 out of the ER into distinct vesicles that colabeled with RD3 and GC1 antibodies in cells cotransfected with RD3 and GC1. $(J-L)$ Intracellular vesicles colabeled with RD3 and Rab 11 antibodies in cells coexpressing RD3 and GC1. $(M-O)$ Distinct intracellular vesicles were labeled with RD3 and ABCA4 antibodies in cells coexpressing RD3 and ABCA4. (Scale bar: $20 \mu \mathrm{m}$.) disk proteins expressed in cultured cells, including ABCA4, peripherin-2, and rom-1 $(22,23)$. These findings support the view that RD3 mediates the membrane trafficking of GC1 out of the ER of cells.

C-Terminal Domain of GC1 Is Required for RD3 Binding. To determine the region of GC1 that contributes to RD3 binding, we expressed GC1 deletion mutants lacking the C-terminal 35 amino acids (M1068), 41 amino acids (M1062), and 48 amino acids (M1055) downstream from the cyclase domain (Fig. $5 A$ ), together with RD3 in HEK293T cells. All mutants were expressed, as revealed by the faint GC1-labeled band in the cell lysate (input lane) of the Western blot. WT GC1, M1068, and, to a lesser degree M1062 coimmunoprecipitated with RD3 on a Rd3-9D12 immunoaffinity matrix, whereas M1055 did not (Fig. 5B). These results suggest that a short conserved segment at 1055-1068 aa downstream of the $\mathrm{GC} 1$ cyclase domain is required for RD3 binding.

\section{Discussion}

In this study, we have identified RD3 as a photoreceptor GCbinding protein required for the stable expression and membrane vesicle trafficking of GC1 and GC2 in photoreceptors and transfected cultured cells. RD3 colocalized with GC1 and GC2 in photoreceptor outer segments and strongly bound to these membrane proteins, as determined by coimmunoprecipitation from detergent-solubilized retinal extracts. The interaction of RD3 with GC1 was confirmed in culture cells expressing these proteins. Deletion mutants indicated that a short segment near the $\mathrm{C}$ ter- minus of $\mathrm{GC} 1$ is required for $\mathrm{RD} 3$ binding to $\mathrm{GC} 1$, although other regions within the cytoplasmic region of $\mathrm{GC1}$ also may contribute to the interaction of RD3 with GC1. RD3 affects the subcellular localization of GC1 in cells. When expressed alone in COS-7 cells, $\mathrm{GC} 1$ is retained in the ER as a stable and functional protein, as demonstrated here and in previous studies (21). Cotransfection of GC1 with RD3 resulted in the export of GC1 from the ER to endosomes, indicating that RD3 plays a crucial role in the early stage of GC membrane vesicle trafficking. In the $r d 3$ mice, no GC1 and GC2 expression was observed by immunofluorescence microscopy, even in the ER of the photoreceptor cells. This suggests that in the absence of RD3, GC1 and GC2 are unstable and rapidly degrade in photoreceptor cells, such that they do not accumulate in sufficient amounts for detection. Because RD3 remains tightly bound to GC1 and GC2 in outer segments of WT mice, as indicated by their colocalization and coimmunoprecipitation from outer segments, RD3 also may serve as an accessory protein required for the vesicle trafficking of GCs from inner to outer segments of rod and cone cells, and also may modulate their enzymatic activity of GCs, although this remains to be determined.

The localization of RD3 in transient transfected COS cells observed in our study is in contrast to the findings of Friedman et al. (10). In their study, overexpression of RD3-GFP resulted in localization of the fusion protein to a subnuclear compartment adjacent to promyelocytic leukemia gene product bodies. We found that untagged overexpressed RD3 localized to endosomes in COS cells. The subnuclear localization of RD3-GFP fusion might result from their use of the large GFP tag, which has been 

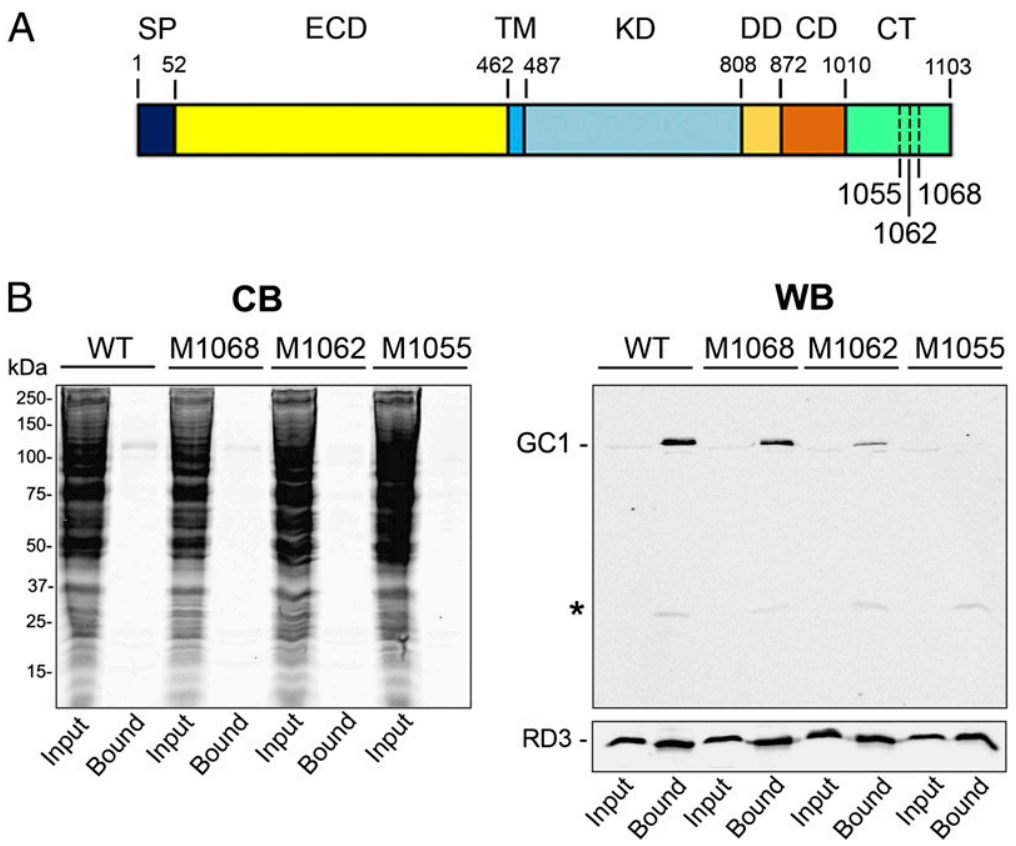

\begin{abstract}
Fig. 5. Binding of RD3 to $W T$ and GC1 C-terminal deletion mutants. (A) A schematic of GC1 domain organization and location of C-terminal deletions. SP, signal peptide; ECD, extracellular domain; $T M$, transmembrane segment; $K D$, kinase domain; DD, dimerization domain; $C D$, cyclase domain; $C T$, C-terminal domain. (B) Coimmunoprecipitation of WT and mutant GC1 with RD3 in HEK293T cells. Detergent-solubilized extracts of cells coexpressing RD3 and WT or GC1 C-terminal deletion mutants (M1068, M1062, and M1055) were immunoprecipitated on a Rd3-9D12 immunoaffinity matrix. The extract (input) and bound fractions were analyzed on Coomassie bluestained gels (CB) and Western blots (WB) labeled for GC1 (Upper) with the GC-2H6 antibody and RD3 (Lower) with the RD3-9D12 antibody. The asterisk indicates the Ig light chain
\end{abstract} that eluted with SDS.

reported to cause abnormal trafficking and subcellular localization of protein in cell overexpression studies (24).

Homozygous $r d 3$ mice exhibit many similarities to GC doubleknockout mice. Both demonstrate a loss of GC1 and GC2 expression, develop shortened and disorganized rod and cone outer segments, undergo progressive retinal degeneration, and display reduced expression and mislocalization of GCAP proteins, presumably due to the loss of GC expression. There are some differences between the two, however. The GC double-knockout mouse has no detectable rod or cone ERG response (15), whereas the $r d 3$ mouse has no cone ERG response, consistent with the absence of GC1, but has a highly attenuated rod response before extensive photoreceptor degeneration occurs (10). The diminished rod ERG response in young $r d 3$ mice might result from a very low level of GC2 expression in rods, as observed in our Western blot analysis. Another interesting difference between the $r d 3$ mouse and the GC double-knockout mouse is in the trafficking of PDE. In GC double-knockout mice, the trafficking of rod-specific PDE (PDE6) is impaired, leading to the suggestion that PDE6 translocation to rod outer segments requires GCcontaining vesicles $(15,25)$. In $r d 3$ mice, however, PDE6 appears to translocate normally to rod outer segments in the absence of detectable GC expression, suggesting the presence of other mechanisms for transporting PDE6 to rod outer segments in 21-dold $r d 3$ mice.

Nonsense and frameshift mutations in the GUCY2D gene encoding GC1 are known to cause LCA type 1 (LCA1), indicating that $\mathrm{GC} 1$ is crucial for vision and viability of human rod and cone photoreceptor cells (2). GC2 cannot compensate for the loss of GC1 in humans. LCA12 patients with a truncation in RD3 exhibit a similar phenotype as LCA1 patients (10), consistent with the loss of GC1 expression as an underlying cause of photoreceptor degeneration. In GC1 knockout mice, however, rod photoreceptors exhibit a reduced photoresponse but remain viable, whereas cones are functionally silent and undergo rapid degeneration $(15,16,26)$. This indicates that rod-specific GC2 can compensate somewhat for the lack of $\mathrm{GC} 1$ in rod photoreceptors of GC1 knockout mice. On the other hand, GC double-knockout mice undergo both rod and cone degeneration similar to that observed in $r d 3$ mice, consistent with the loss of GC1 and GC2 expression as the cause of photoreceptor degeneration in $r d 3$ mice. A frameshift mutation in the gene encoding RD3 in the $r c d 2$ collie (12) is also likely to produce an unstable, nonfunctional RD3 protein, resulting in the loss of GC expression and trafficking as well as rod and cone degeneration.

Why does the loss in GC expression cause photoreceptor degeneration? As discussed by Fain (27), photoreceptors, like other neurons, are designed to function and survive over a wellcontrolled concentration of intracellular $\mathrm{Ca}^{2+}$. Excessively high or low $\mathrm{Ca}^{2+}$ levels can kill photoreceptors. In photoreceptor outer segments, calcium homeostasis is achieved by the influx of $\mathrm{Ca}^{2+}$ through cGMP-gated channels and the balanced efflux of $\mathrm{Ca}^{2+}$ through the $\mathrm{Na} / \mathrm{Ca}-\mathrm{K}$ exchanger. A loss of $\mathrm{GC}$ expression and trafficking impairs the ability of photoreceptors to synthesize cGMP in their outer segments. As a result, the cGMP-gated channels remain in their closed state. We speculate that constitutive closure of the cGMP-gated channels, together with the continued extrusion of intracellular $\mathrm{Ca}^{2+}$ by the $\mathrm{Na} / \mathrm{Ca}-\mathrm{K}$ exchanger, may cause a drop in $\mathrm{Ca}^{2+}$ levels inside outer segments to below a critical level required for long-term photoreceptor survival. Thus, the mechanism of photoreceptor degeneration in LCA1 2 patients and $r d 3$ mice would be similar to that for LCA1 patients and GC double-knockout mice and may result from the loss of cGMP production and prolonged reduction in intracellular $\mathrm{Ca}^{2+}$ level. Another possible mechanism for photoreceptor degeneration caused by RD3 deficiency may be ER stress associated with the mistrafficking of GC in photoreceptors. However, the finding of apparently similar photoreceptor degeneration in GC double-knockout mice and $r d 3$ mice suggests that ER stress is not the primary mechanism of photoreceptor cell death. Our findings indicating that RD3 is crucial for GC expression and trafficking in photoreceptors provides a basis for developing therapeutic strategies for treating these retinal degenerative diseases.

\section{Materials and Methods}

cDNAs and Antibodies. Full-length human and mouse RD3 CDNAs were generated by PCR using retinal CDNAs as templates. Human RD3 was cloned into pcDNA3 (Invitrogen). The human GC1 CDNA in pRcCMV was a generous gift from Dr. Alexander Dizhoor (Pennsylvania College of Optometry, Elkins Park, PA). Three GC1 deletion mutants (M1068, M1062, and M1055) were generated by introducing a stop codon after amino acids 1068, 1062, and 1055 , respectively. All of the constructs were verified by DNA sequencing. 
The Rd3-9D12 monoclonal antibody was generated from mice immunized with an affinity-purified GST-fusion protein containing the C-terminal 41 aa of mouse RD3, as described previously (28). Polyclonal antibodies were generated against a 15-aa C-terminal peptide of mouse RD3 and purified on a peptide affinity column and nitrocellulose blots of GST-RD3-C-terminal fusion peptides, as described previously (14). Monoclonal antibodies to CNGA1 (PMc-1D1), GC1 (GC1-2H6), ABCA4 (Rim-3F4), rhodopsin (Rho-1D4), and peripherin-2 (Per-5H2) have been described previously (29). GC1 and GC2 polyclonal antibodies were a generous gift from Dr. David Garbers (University of Texas Southwestern Medical Center, Dallas), GCAP1 and GCAP2 antibodies were kindly provided by Dr. Wolfgang Baehr (University of Utah, Salt Lake City), PDE- $\alpha$ and cone arrestin antibodies were obtained from Affinity BioReagents, and $r d 3$ mice (4Bnr, albino strain) were obtained from The Jackson Laboratory. All animal protocols were approved by the University of British Columbia's Animal Care Committee.

Coexpression of RD3 and GC1 or ABCA4. HEK293T cells were cotransfected with $10 \mu \mathrm{g}$ of the human RD3 plasmid and $10 \mu \mathrm{g}$ of either human $\mathrm{GC} 1$ or human ABCA4 plasmid using the calcium phosphate procedure. At $48 \mathrm{~h}$ after transfection, the cells were solubilized in $1 \%$ Triton X-100 in PBS containing complete protease inhibitor for $1 \mathrm{~h}$ at $4{ }^{\circ} \mathrm{C}$ with stirring. After the insoluble material was removed by centrifugation, the supernatant was used for immunoprecipitation studies.

Mouse Retinal Membrane Preparation, Immunoprecipitation, and Mass Spectrometry. A membrane fraction from $40-80$ retinas of 21 -d-old WT and $r d 3$ mice were prepared as described previously (30). In brief, for immunoprecipitation studies, a mouse retinal membrane extract ( $\sim 3.5 \mathrm{mg}$ of protein) was incubated in hypotonic buffer [ $20 \mathrm{mM}$ Hepes $(\mathrm{pH} 7.4)$ containing complete protease inhibitor) for $20 \mathrm{~min}$ on ice and then solubilized with $700 \mu \mathrm{L}$

1. den Hollander Al, Roepman R, Koenekoop RK, Cremers FP (2008) Leber congenital amaurosis: Genes, proteins and disease mechanisms. Prog Retin Eye Res 27:391-419.

2. Perrault I, et al. (1996) Retinal-specific guanylate cyclase gene mutations in Leber's congenital amaurosis. Nat Genet 14:461-464.

3. Luo DG, Xue T, Yau KW (2008) How vision begins: An odyssey. Proc Natl Acad Sci USA 105:9855-9862.

4. Lowe DG, et al. (1995) Cloning and expression of a second photoreceptor-specific membrane retina guanylyl cyclase (RetGC), RetGC-2. Proc Nat/ Acad Sci USA 92:5535-5539.

5. Sung $\mathrm{CH}$, Tai AW (2000) Rhodopsin trafficking and its role in retinal dystrophies. Int Rev Cytol 195:215-267.

6. Tam BM, Moritz OL, Hurd LB, Papermaster DS (2000) Identification of an outer segment targeting signal in the $\mathrm{COOH}$ terminus of rhodopsin using transgenic Xenopus laevis. J Cell Biol 151:1369-1380.

7. Deretic D (2006) A role for rhodopsin in a signal transduction cascade that regulates membrane trafficking and photoreceptor polarity. Vision Res 46:4427-4433.

8. Chang B, Heckenlively JR, Hawes NL, Roderick TH (1993) New mouse primary retinal degeneration (rd-3). Genomics 16:45-49.

9. Linberg KA, Fariss RN, Heckenlively JR, Farber DB, Fisher SK (2005) Morphological characterization of the retinal degeneration in three strains of mice carrying the rd-3 mutation. Vis Neurosci 22:721-734.

10. Friedman JS, et al. (2006) Premature truncation of a novel protein, RD3, exhibiting subnuclear localization is associated with retinal degeneration. Am J Hum Genet 79 1059-1070.

11. Lavorgna G, et al. (2003) Identification and characterization of C1orf36, a transcript highly expressed in photoreceptor cells, and mutation analysis in retinitis pigmentosa. Biochem Biophys Res Commun 308:414-421.

12. Kukekova AV, et al. (2009) Canine RD3 mutation establishes rod-cone dysplasia type 2 $(\mathrm{rcd} 2)$ as ortholog of human and murine rd3. Mamm Genome 20:109-123.

13. Kwok MC, Holopainen JM, Molday LL, Foster LJ, Molday RS (2008) Proteomics of photoreceptor outer segments identifies a subset of SNARE and Rab proteins implicated in membrane vesicle trafficking and fusion. Mol Cell Proteomics 7:1053-1066.

14. Illing M, Molday LL, Molday RS (1997) The 220-kDa rim protein of retinal rod outer segments is a member of the ABC transporter superfamily. J Biol Chem 272:10303-10310.

15. Baehr W, et al. (2007) The function of guanylate cyclase 1 and guanylate cyclase 2 in rod and cone photoreceptors. J Biol Chem 282:8837-8847.

16. Coleman JE, Zhang Y, Brown GA, Semple-Rowland SL (2004) Cone cell survival and downregulation of GCAP1 protein in the retinas of GC1 knockout mice. Invest Ophthalmol Vis Sci 45:3397-3403. of $1 \%$ Triton $\mathrm{X}-100$ in $20 \mathrm{mM}$ Hepes (pH 7.4) and $0.3 \mathrm{M} \mathrm{NaCl}$. After stirring on ice for $1 \mathrm{~h}$, the solution was centrifuged at $67,000 \times g$ for $10 \mathrm{~min}$ to remove unsolubilized material. Then the detergent-solubilized retinal extract was incubated with $75 \mu \mathrm{L}$ of immunoaffinity matrix consisting of either the Rd3-9D12 or GC1-2H6 monoclonal antibody directly coupled to CNBractivated Sepharose (GE Health Bio-Sciences) (14). The unbound fraction was retained, and the bound protein was eluted from the matrix with $4 \%$ (wt/ vol) SDS in PBS. The fractions were analyzed by SDS/PAGE and Western blot analysis as described previously (30). The immunoprecipitated protein fraction was prepared for LC-MS/MS as described previously (30).

Immunofluorescence Microscopy. Retinal cryosections from 21-d-old homozygous $\mathrm{rd} 3$ and WT mice and COS-7-transfected cells were labeled as described previously $(23,30)$. Antibodies used for these studies included rabbit anti-calnexin antibody (Sigma-Aldrich) diluted 1:200, mouse anti-rab11 monoclonal antibody (BD) diluted 1:25, GC-2H6 hybridoma fluid diluted 1:5 (29), and rabbit anti-Rd3 antibody diluted 1:250 (this study). Samples were counterstained with DAPI nuclear stain and visualized under a $40 \times$ objective with a Zeiss LSM 700 confocal scanning microscope equipped with ZEN 2009 image analysis software. Photographs were compiled with Photoshop CS (Adobe Systems) without digital enhancement.

ACKNOWLEDGMENTS. We thank Dr. Alexander Dizhoor (Pennsylvania College of Optometry, Elkins Park, PA) for the human GC1 CDNA, Dr. Wolfgang Baehr (University of Utah, Salt Lake City) for the GCAP1 and GCAP2 antibodies, and Dr. David Garbers (University of Utah, Salt Lake City) for the GC1 and GC2 antibodies. This work was supported by grants from the National Eye Institute (EY02422), Canadian Institutes for Health Research/ Foundation Fighting Blindness (Canada) (CIHR RMF-92101), and the Macula Vision Research Foundation.

17. Dizhoor AM, Lowe DG, Olshevskaya EV, Laura RP, Hurley JB (1994) The human photoreceptor membrane guanylyl cyclase, RetGC, is present in outer segments and is regulated by calcium and a soluble activator. Neuron 12:1345-1352.

18. Yang RB, Garbers DL (1997) Two eye guanylyl cyclases are expressed in the same photoreceptor cells and form homomers in preference to heteromers. $J$ Biol Chem 272:13738-13742.

19. Sokal I, et al. (2000) $\mathrm{Ca}(2+)$-binding proteins in the retina: From discovery to etiology of human disease(1). Biochim Biophys Acta 1498:233-251.

20. Olshevskaya EV, Ermilov AN, Dizhoor AM (2002) Factors that affect regulation of CGMP synthesis in vertebrate photoreceptors and their genetic link to human retinal degeneration. Mol Cell Biochem 230:139-147.

21. Peshenko IV, Olshevskaya EV, Dizhoor AM (2008) Binding of guanylyl cyclase activating protein 1 (GCAP1) to retinal guanylyl cyclase (RetGC1): The role of individual EF-hands. J Biol Chem 283:21747-21757.

22. Goldberg AF, Moritz OL, Molday RS (1995) Heterologous expression of photoreceptor peripherin/rds and Rom-1 in COS-1 cells: Assembly, interactions, and localization of multisubunit complexes. Biochemistry 34:14213-14219.

23. Zhong M, Molday LL, Molday RS (2009) Role of the C terminus of the photoreceptor $A B C A 4$ transporter in protein folding, function, and retinal degenerative diseases. J Biol Chem 284:3640-3649.

24. Lisenbee CS, Karnik SK, Trelease RN (2003) Overexpression and mislocalization of a tail-anchored GFP redefines the identity of peroxisomal ER. Traffic 4:491-501.

25. Karan S, Frederick JM, Baehr W (2010) Novel functions of photoreceptor guanylate cyclases revealed by targeted deletion. Mol Cell Biochem 334:141-155.

26. Yang RB, et al. (1999) Disruption of a retinal guanylyl cyclase gene leads to conespecific dystrophy and paradoxical rod behavior. J Neurosci 19:5889-5897.

27. Fain GL (2006) Why photoreceptors die (and why they don't). Bioessays 28:344-354.

28. MacKenzie D, Molday RS (1982) Organization of rhodopsin and a high molecular weight glycoprotein in rod photoreceptor disc membranes using monoclonal antibodies. J Biol Chem 257:7100-7105.

29. Poetsch A, Molday LL, Molday RS (2001) The CGMP-gated channel and related glutamic acid-rich proteins interact with peripherin-2 at the rim region of rod photoreceptor disc membranes. J Biol Chem 276:48009-48016.

30. Molday LL, Wu WW, Molday RS (2007) Retinoschisin (RS1), the protein encoded by the $\mathrm{X}$-linked retinoschisis gene, is anchored to the surface of retinal photoreceptor and bipolar cells through its interactions with a $\mathrm{Na} / \mathrm{K}$ ATPase-SARM1 complex. J Bio/ Chem 282:32792-32801. 